\title{
A New Case of a Disseminated Extragenital Bullous Lichen Sclerosus
}

\author{
H.Amarouch, S.Maouni, H.Zaouri, L.Benzekri, B.Hassam
}

Dermatology department, university hospital of IBN SINA Rabat Morocco

\begin{abstract}
:
Introduction: Lichen sclerosus atrophicus (LSA) is a chronic inflammatory skin disease of unknown etiology. it is characterized by a severe itching, skin atrophy and sclerosis. It mainly affects the anogenital area of postmenopausal women [1]. The presence of an isolated Extragenital form in its bullous variant is rarely described.
\end{abstract}

Case report: Herein we report a case of a 61 years old woman with a history of 8months Vesicular and bullous itching lesions disseminated to the trunk and arms with no oral or genital lesion was noted. Histology finding confirmed the diagnosis of a bullous extra genital LSA. She responded very well to oral steroids.

Conclusion: bullous extra genital LSA is a diagnosis to consider in front of all blisters diseases. It s rarity is possibly due to misdiagnosis and underreporting.

Keywords: steroids, disseminated blisters, lichen sclerosis et atrophicus, non-genital, painful, pruritus

\section{INTRODUCTION}

Lichen sclerosus atrophicus (LSA) is a chronic inflammatory skin disease of unknown etiology.it is characterized by a severe itching, skin atrophy and sclerosis. It mainly affects the anogenital area of postmenopausal women [1]. The presence of an isolated Extragenital form in its bullous variant is rarely described. Herein we report a new case.

\section{CASE REPORT}

A 61 years old female, presented with a history of 8 months Vesicular and bullous itching lesions, localized on the trunk, arms and forearms. In the course of healing, these lesions leave in place a sclerosal and atrophic white patches. Clinical examination revealed several hypopigmented sclerosal and atrophic patches, ranging in size from 5 to $10 \mathrm{~cm}$, along with Vesiculobullous lesions and crusted erosions involving her trunk, arms, forearms, and medial thighs [Figure 1]. No oral or genital lesion was noted. Histological findings objectified a subepidermal blister with a thinning of the epidermis with compact hyperkeratosis, basal apoptosis, and a band of homogenized collagen in the papillary dermis above a varying degree of lymphocytic infiltrate[Figure 2]. Direct immunofluorescence was négatif. The patient received an oral prednisone $0.5 \mathrm{mg} / \mathrm{kg} /$ day with a total improvement of the lesions and pruritus after 6 weeks of treatment.

\section{DISCUSSION}

LSA was initially described by Hallopeau in 1887 [1]. It is an inflammatory, mucocutaneous disorder that affects male and especially female with a debilitating impact on the quality of life. Common localization is the anogenital area. [1] The incidence of extra-genital LSA is difficult to assess, because of the fuzzy boundary with morphea, underreporting, and the absence of functional symptoms in most cases. However, it is estimated that $15-20 \%$ of anogenital LS also involve extra-genital areas, with only $2.5 \%$ patients presenting an isolated extra-genital LSA [2]. As for the bullous variant, only 6 cases were reported in the literature. The etiology of LSA remains poorly understood .Several factors have been implicated, including genetic susceptibility, hormonal disorders, chronic infections and injuries [3]. However, the autoimmune theory remains the most plausible, it was confirmed by the demonstration of an Igg antibodies directed against the extracellular matrix in $74 \%$ of group LSA against $7 \%$ in the control group, as well as the presence of the basement membrane antibodies of the bullous pemphigoid. [3] Genital LSA is manifested by a progressive atrophy with secondary sclerotic scars and severe itching, bleeding and pain which leads to serious functional sequelae. [4]The extra- 


\section{H.Amarouch et al.}

genital LS is usually asymptomatic and preferentially involves the neck, shoulders, trunk and proximal ends. [4] Clinically, as in our patient the first vesicles and bullous lesions healed by leaving in place a white ivoiry, sclerosal and atrophic plaques [4].Histologically, in the first stages, there is a lymphocytic infiltrate in the dermo-epidermal junction with keratinocyte necrosis as well as a parakeratosis and epidermal atrophy. The evolution is marqued by an Homogenization of the collagen with a major edema responsible for an elastin fibers destruction in the papillary dermis. Histologically, the formation of the bubble was explained by two mechanisms: either the vacuolar interface dermatitis gives a degeneration of the MB and then the blisters; or that the importance of the papillary dermis edema is responsible for an instability of the architecture of the collagen fibers with blister formation especially with the intensity of the epidermal atrophy[5].Although there is no definitive or satisfactory treatment for LSA, very strong topical corticosteroids remain the treatment of choice. Many treatment modalities have been used with varying degrees of responses, including topical and oral steroids or topical and systemic vit A, the vit D analogues, topical calcineurin inhibitors, phototherapy, and surgery. [6]

\section{CONClusion}

Bullous extra genital LSA is a diagnosis to consider in front of all blisters diseases. It $\mathrm{s}$ rarity is possibly due to misdiagnosis and underreporting.

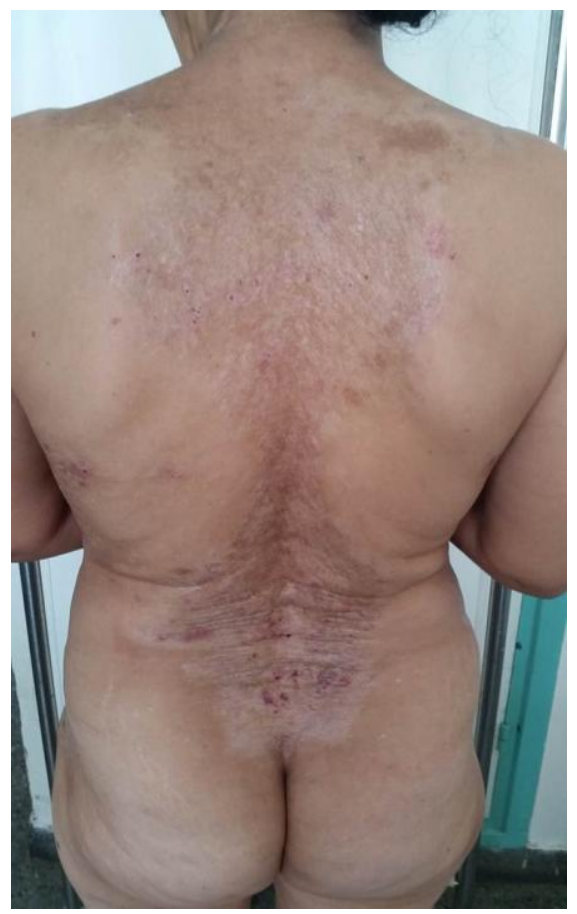

Figure1. Post bullae erosion within pre-existing atrophic lesions involving her back

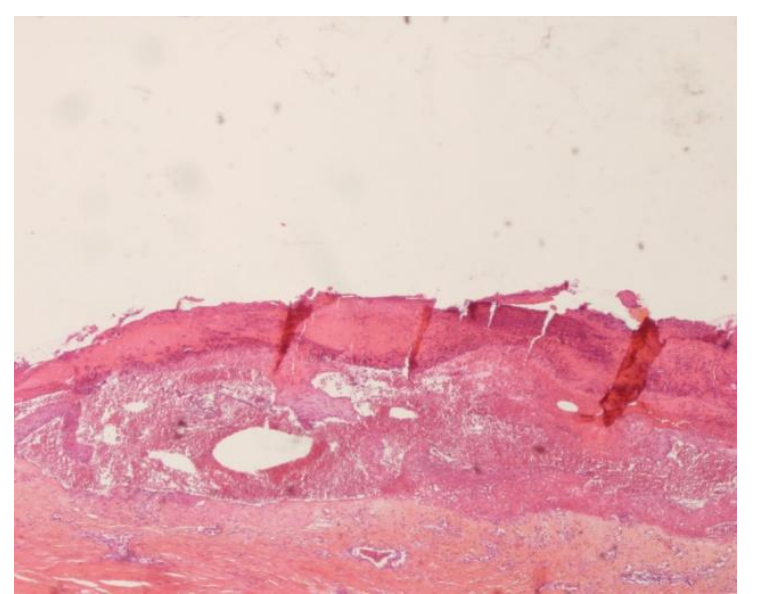

Figure2. HE stain $\times 40-A$ subepidermal blister with a thinning of the epidermis with compact hyperkeratosis, basal apoptosis, and a band of homogenized collagen in the papillary dermis above a varying degree of lymphocytic infiltrate 


\section{REFERENCES}

[1] Smith YR, Haefner HK. Vulvar lichen sclerosus : pathophysiology and treatment. Am J Clin Dermatol. 2004; 5(2):105-25.

[2] Kreuter A AND AL. Coexistence of lichen sclerosus and morphea: A retrospective analysis of 472 patients with localized scleroderma from a German tertiary referral center. J Am Acad Dermatol. 2012;67:1157-62

[3] Meyrick Thomas RH and al Lichen sclerosus et atrophicus and autoimmunity: A study of 350 women. Br J Dermatol. 1988;118:41-6

[4] Ballester I and Al Extragenital bullous lichen sclerosus atrophicus. Dermatol Online J. 2009; 15:6.

[5] Kowalewski $\mathrm{C}$ and al Alterations of basement membrane zone in bullous and non-bullous variants of extragenital lichen sclerosus. Am J Dermatopathol. 2004;26:96-101

[6] Neill SM and al Dermatologists BAo. British Association of Dermatologists' guidelines for the management of lichen sclerosus 2010. Br J Dermatol. 2010;163:672-682 